\title{
Register
}

\section{Personen-, Orts- und Sachregister}

Abgar 179

Admedera 34

Administration, römische $\quad 135,139$ 140

Africa 81, 93-97, 120

Agrippa II. 142-143

Ägypten 190-191

Akoris, Ägypten 35

Albinus, Rufius 78

Alexandria 58, 71, 77, 215

Alypius 211

Ambrosius 75, 77, 133-134, 145147

Ambrosius von Mailand 48

Ammaedara 38

Ammianus Marcellinus 25, 32, 36, 70

Anonymus de rebus bellicis $\quad 62-63$

Antinoopolis, Ägypten 37

Antiochia, Syrien 36, 45, 58, 171174, 176-177

Antiochos IV. 144

Antoninus von Fussala 211

Apamea 173

Aphrodisias 192, 195

Aphrodite-Kult 13

Apollodorus, procos. Africae 80

Aquileia 134-135

Arabia 33

ARCADIUS 80, 107, 172

Archäologie 187-196

Asklepiodotos, PPO 83

Asterius, comes Orientis 79

Aufruhr 107, 120-121, 124
Augustinus $211,49,95,99,121$, 125

AURELIAN 21, 22, 46

Babylas 175-176

Baiae, Vespasian-Tempel 35

Baitokaikai, Phoenikien 34

Beamte, kaiserliche 209-215

Beroia 166

Bischof, Bischöfe $\quad 70,81,94-96,99$, 104,109, 117-120, 122-123, 128, $145,149,162,175,211-215$

Breno, Minerva-Tempel 193

Brixia 21

Cabassa, Narbonensis 28

Caesarea, Mauretania 26

Caesarius, PPO 79

Calama 213

Capua 36

CARINUS 21-22

CARUS 21-22

Chester, Anglia 26

Christianisierung 203, 97-98, 116117, 160-161, 165, 174, 178

Chronicon Paschale 64

Cingulum, Picenum 21

Cirta, Numidia 25

Claudius Gothicus 21, 29

Codex Theodosianus 75, 96, 98,

$111,117,170,187,201-210$

Comes orientis 133,146

Constantius I. 22-23, 26-27

COnstantius II. 27-28, 67-69, 71, 109, 176-177, 205-206

CRISPUS 165 
Cynegius Maternus, PPO 77, 122, $160,163,166-171,178,213-214$

Cyprian von Carthago 41

Damasus I., Papst 75

damnatio memoriae 20-24, 31, 34, 36,45

Daphne 122, 126

Daphne, Apollon-Tempel 175, 177178

DECIUS 21

Dendera 191

Desakralisierung 207-209

DIOKLETIAN 21-24, 44

Donatisten 93-95

Dura Europos 188

Durostorum, Moesia Inf. 30, 44

Dyrrachium 23

St. Dasius 30, 41, 44

Edessa 73

Edessa, Osrhoene 166, 178-180

Cunctos populos, Edikt 205

Egeria 179

Eigentumsübertragung $55,59,62$, $64,71,80$

Eleusis 51

Emesa 173

Entsakralisierung $\quad 59,69,71,177$

Ephesus 35, 51, 190

- Hadrian-Tempel 35

Eretria, Euboea 31, 50

Eugenius 78-7

Eunapios 102

Eusebius von Caesarea 7-18, 19, 23, $26,29,41-43,45,47,55-58,60-$ $63,100,179,189$

- Tricennalienrede 55, 58, 65

- Vita Constantini 55,60-61

FAUSTA 165

Festwesen 112-113

Firmicius Maternus 61-63

Flavianus, Bischof

von Antiochia 175-176

Flavius Josephus 142-143

FLORIANUS 20

Frigidus, Schlacht am 74, 79
GALERIUS 23, 24, 26-27, 29, 31

GALLIENUS 21, 34

GALLUS 21

Gaza 173, 212

generalitas von Gesetzen 205-206

Gerasa 33

Gesetzgebung, Eigenart

- spätantike 209-216

- anti-häretische 90,152

- anti-pagane 9-10, 15, 76-82, 93-94, 99, 103, 108-109, 111, 152, 192, 201-209, 213-216

Gewalt 162-165, 167-168, 171$172,174-175,178$

Gewalt gegen Kultorte 133-136

Gewalt(anwendung), religiös motiviert 133-153

Gewaltdiskurs 145-148

Gigithi, Byzancena 25

Grabeskirche 66

Gracchus, Furius

Maecius, PV 75

GRATIAN 24-25, 75-76, 82

Gregor von Nazianz 70

Häretikergesetze 99,152

Harpinium 28

,Heidentum“ (Begriff) 9

Heliopolis-Baalbek 13, 173

Herrscherkult 19-25, 29-39, 44-46, $48-50,60-61,83$

Hieronymus 48, 65

Hilarius, Bischof

von Poitiers $62,65,71$

Hispellum 29-30, 37, 60

Historia acephala 71

Historiographie, christliche 117, 206

HONORIUS $67,75,79,82,93,95$, $100,107,113,116,120$

Hypatia 83

Jerusalem, Grabeskirche 12-13

Johannes Chrysostomos 175-176, 187

JOVIAN $24,36,72,177$

Judäa 141-142

1. Jüdischer Aufstand 141-144 
jüdische Elite 141-143

JULIAN 24, 32, 71-73, 100, 104, 111, 118, 161, 165-166, 175, 177-179, 204

JUSTINIAN 19,187

Kaiserkult 15-16, 160

Kaisertum 159-160, 169

Kaisertum, christliches 146-152

Kallinikon 133-136, 145-147

Karrhai 173,179

Karthago 81, 93-94

Kirche, nordafrikanische 211-214

Kirchenbau 66, 74

Konfiskation 160-161, 165, 207

Konflikt, religiöser 93-97, 124, 133-134, 145, 148-153

KONSTANTIN 7-18, 24, 26-29, 32, 45, 47-48, 55-67, 100, 111, 125, 148, 159-160, 165, 170, 176, 188-189, 205

- Religiosität 16-17

Konstantinopel 14, 58, 65, 66-67

Konversion 165

Koptos, Ägypten 32

Kultgegenstände 82

Kultstatuen 58

Kultstatuen (pagane) 9, 14-15

Kyrene 126, 193

Kyrill von Alexandria 211

Laktanz 23, 29, 32, 36

Lambaesis, Numidia 22

Lampadius, C. Ceionius Rufius Volusianus 74

Laterankirche, Rom 66

LEO I. 109

Leon, Gallaecia 42

Libanios 58, 124-127

Libanios, Epitaphios 71-72

Libanios, Pro Templis $\quad 69,76,111$, 162-180

Luna 189

Madalianus, L.Crepereius 65

Makarios von Jerusalem 66

Makkabäer 143-144

makkabäische Brüder 133
Mamertinus, PPO 73

Mamre 10-12

Manichäer 99

Marcellus von Apameia 109, 213

Marcellus, Märtyrer 42-43

M. Iulius Philippus 21

Marinus, Märtyrer 41

Markos von Arethusa 70-71

Marnas-Tempel, Gaza 107, 121, 193-194

Martin von Tours 187

Märtyrerakten 40-42

Maßnahmen, anti-pagane 7-18

MAXENTIUS 23-24, 29

MAXIMIANUS 22-25, 28,36

MAXIMINUS DAIA 26, 29

Medinet Habu 191

Meletius, Bischof 176

Meron, Phrygia Salutaris 34

Mesopotamia 32

Moesia Inferior 22

Mönche 70, 133-134, 163-164, $168,172,174-176,209$

Münzprägung 62-64

Nicomachus Flavianus,

Virius 74,78

Nicomedia 32

Nikopolis, Moesia inf. 35

Nizäner 94-95, 97, 99

Normen, imperiale 135, 139

notarii 213-214

NUMERIANUS 21-22

officiales 100, 103- 104, 113-115, $120,122-123,125,210$

Opfer, Opferverbot $\quad 9-12,15-16,56$, $60,67,76-77,161,164,166-$ 167, 206-207

Oriens, Diözese 172

Origines 19

Origines, Contra Celsum 45-46

Palladius, dux Osrhoenae 73

Panopolis 201

parabalani 211

Passio Iuli veterani 43

Pax Romana 135 
St. Peter, Rom $\quad 66,188$

Philae 187

Philippopolis 33

PHILIPPUS ARABS 19-20, 21, 33, 46

Philostorgius 37,48

Pionius, Märtyrer 41

Plutarch 140

Porphyrios von Gaza 93-94, 104, $107,109,114,119,121,126$

Porphyrius von Tyros 30

Praetextatus, Vettius Agorius 74, 100,210

Priester 38-39, 49

privilegium 101, 108, 119

Probus 20

Proclianus, PPO 79

QUIETUS, Usurpator 32-33

Ratsherren $172,176-178$

Ravenna 211

Reichsverwaltung 209-212

Religionsgesetzgebung 93-97, 103109, 115-116, 124-125, 161, $170,173,201-216$

Religionspolitik, kaiserliche 59-62, 69-73,75, 79-84, 97-101, 111$112,202-208,215-216$

res privata $59,62,64-66,71-72$, $75,76,80,82-83$

Restauration, heidnische $71-72,74$, $78,161,178$

Rom 58, 65, 66, 69, 73, 74, 77, 165, 189,194

ROMULUS 27

Rufinus von Aquileia 49, 215

Saint-Aubin-sur-Mer 194-195

SALONINUS, P. Licinius 21

Santa Prisca, Rom (Mithräum) 194

Sarsina 195

Schenute von Atripe $103,124,187$, 201-202, 216

Schutz heidnischer Kulte 73-74

Schutz von Tempeln 112

Serapeion, Alexandria 77, 111, 215

Sibyllinische Bücher 82

Sirmium 27
Smyrna, Kaisareion 35

Sokrates, Kirchenhistoriker 61

Sozomenos, Kirchenhistoriker 71, $116,206,213-215$

städtische Elite 159-162, 173

stasis 151

Statthalter 93, 96-104, 107-108, 111-114, 117-118, 120-

$128, \quad 208,210-213$

Statuen $35-37,48,188-191$

Stilicho 82, 95

Strafmaß 106-107, 109-110, 124

Strafprozesse 108, 112-113

suggestiones 211

Symmachus, Q. Aurelius 73, 74, 75

Synagoge(n), Zerstörung 133-136

Synesios 125-126, 128

Syria Coele, Provinz 173, 175

Tatianus, Fl. Eutolmius, PPO 37, 163

Tempel 30-38, 44, 55-61, 164-165, 188-192, 205

- Bewahrung 69-70, 73, 77, 79$81,166,207$

- Plünderung 70-71, 77, 79, 160, 172

- Schließung 55, 67, 71, 75, 160, 188, 191-192, 207-208, 213

- Umnutzung 62, 68, 70,81, 177, 191, 193, 195-196, 207

- Verlassen 189, 191-192, 195196

- Zerstörung 7, 9, 56-57,6870,71-72, 75, 77, 79-84, 160, 163-164, 166-168, 171-172, 174, 178-179, 187, 193-195, 207-209, 212-215

Tempelgut $55,58-59,63-64,70$ $72,78-80$

Tempelprostitution 13-14

Tetrarchie 22-24

Thamugadi, Numidia 21, 38

Theodoret 174-175, 213 
Theodosius I. 19, 25, 49, 75-76, 79, 101, 108-111, 116, 133-136, 145-148, 161-162, 163-165, 166-167, 169-171, 172-173, 176-178, 192, 205-207

TheOdosius II. $\quad 82-83,102,201-$ 202

Theophilos, Bischof

von Alexandria 77, 215

Thessaloniki 24

Thuburuscum, Numidia 21

Tiberius Alexander 141

Tieropfer s. Opfer

Tingis, Mauretania 42

Trajansforum 28

Trebonianus Gallus 21

Troesmis, Scythia Minor 28
Unterdrückung paganer Kulte 9798, 100-101, 105-107, 110, 112, $118,121-122$

VALENS 25, 72-73,161, 210 170, 176

VALENTINIAN I $24-25,72-73,170$, 204, 210

VALENTINIAN II. $\quad 75,83$

VALERIAN 21, 34

Vegetius 30

Verecundia 22

Vergöttlichung 21-30,33, 40

Verona, Capitolium 192

Vesta-Kult 75-76

Vicetia 28

Victoria-Altar 69,75

Vita Porphyrii 212-213

Zosimos 10

\section{Quellenregister}

Literarische Quellen

Acta Agap. 3105

Acta conc. oec. IV 1: 155104

Acta Marcelli, rec. M 142

- N 343

Acta procos. S. Cypr. 142

Afric. chronogr., frg. 1810

Ambr. ep. 74 (40) 133-136, 145148

Ambr. ep. extr. coll. 1 133-134

Ambr. hexaem. 6,9,57 49

Amm. 23,5,7-8 32

- 25,10,1-2 36

- 30,7,2 25

Anon. de rebus bell. 2,1f. 63

- 2,4 63

Aug. civ. XVIII 54120

Aug. ep. 91,8 94

- 232,1 98

Chron. Pasch. ad a. 32564

Epikt. diss. 4,1,14 45

Eun. F 29 Blockley 177
Eun. v. soph. $503 \quad 102$

Eus. d. e. 5,9,7-8 12

Eus. h. e. 8,1323

Eus. 1. C. $7,13 \quad 55$

- 8,2-3 58

- 8,313

- 8,5-7 13-14

Eus. mart. Pal. 3,1 44

Eus. v. C. 1,21 26

- $2,45,19$

- 2,46,3 66

- 2,48-60 61

- $3,1,5 \quad 57$

- 3,26,2 13

- $3,52 \mathrm{f} .12$

- 3,53,2 9

- $3,54,1$ f. 7

- 3,54189

- $3,54,214$

- $3,54,314$

- 3,56-58 57 
- 3,58,3 13

- $4,1615,61$

- 4,1947

- 4,25 14

- 4,25,1 56

- 4,39,2 7

- 4,60 16

Firm. err. prof. rel. 28,5 62

- 28,6 62

Hieron. comm. in Ier. VI 18,6 11

Hieron. chron. ad a. 33165

Hieron., exeg. in Dan. 3,18 48

Hilar. ep. ad Const. 1, $6 \quad 62$

Itin. Egeriae 19,2-17 180

Jo. Chrys. de S. Babyla 67-113 175

Jo. Chrys. hom. 1-8 adv. Iud. 176

Jo. Chrys. hom. 4,6 in 1. Cor. (PG 61, 38-40) 176

Jo. Chrys. hom. 15,5 in Phil. (PG 62, 295) 165

Jo. Mal. chron. 13,13 14

Joseph. bell. 2,361-365 142-143

Lact. mort. pers. 19,2 32

- 4223

Lib. ep. 958171

Lib. or. 12,69 169

- 12,79-82 169

- 18,23111

- 18 (Epit.), 126 71-72

- 30 (Pro Templis) 162-180

- 30,7 161

- $\quad 30,10169$

- 30,3710

- 30,52-53 160-170

- 50,36169

- 62,859
1Makk. 3,16-23 144

Mart. Das. 731

- 7-8 44

Mart. Polyc. 839

M. Diac. v. Porph. 69193

- 75-79 193

Orig. c. Cels. 8,65 45

- 8,6646

- 8,6846

- 8,7346

Paneg. X (II) 10,6,5 37

- $\quad$ XI (III) 2,4 23, 26

Passio S. Marini = Eus. h. e. 7,15 41

Passio S. Pionii $8 \quad 41$

Passio SS. Scillit. $3 \quad 40$

- 6,9 40

Philost. h. e. $2,17 \quad 15$

Plin. ep. 10,34,1 138-139

Plut. mor. 813d-f 140

Porph. De abstin. 2,35,40-43 30

Prok. bell. Pers. 2,12,26 179

- $1,19,37187$

Quodvultd. I prom. 13,15 125

Schenoudi, Oeuvres (ed. É. Amélineau), t. II, fasc. 3, 1914, p. 523,7201

Socr. h. e. $1,18,1 \quad 61$

Soz. h. e. $2,4,3 \quad 11$

- 7,12,12 116

Thdt. h. e. 4,24,2 111

- 5,21,5 213

Veget. mil. 2,5 30

Zos. 2,31 14

- 4,3,2-3 161, 210

- 4,3,3 102

Gesetzestexte

Cod. Iust. 4,35,23-24 50

Cod. Theod. 1,1,5 pr. 204

- 9,16,7 161

- 9,16,9 204

- $15,1,3679,173$

- $16,1,173$
- $\quad 16,10,2 \quad 10,205-206$

- $16,10,3 \quad 68,207$

- 16,10,4 67

- $16,10,8 \quad 166,169$

- $16,10,1278$

- $16,10,15 \quad 80,81,207$ 
- $\quad 16,10,16 \quad 80,216$

- $16,10,17217$

- $16,10,1981,82$

- $16,10,20 \quad 82$

- $16,10,21 \quad 102$

- $16,10,2483$

$$
\text { - } \quad 16,10,25 \quad 84,203
$$

Const. Sirmond. 12 93, 94, 109, $113,117,120,123,213$

- 14 95, 96, 103, 107, 114, 115, 120,213

Nov. Theod. 3,8 103, 110

\section{Inschriften}

CIL 3,7586 21

CIL 5,3332 192

CIL 8,2387 38

CIL 8,2403 38

CIL 8,7034 38

CIL 8,10516 38

CIL 8,17896 38

CIL $115265=$ ILS $705 \quad 15,101$

IG *II 2,5206 39

IGR 1,1181 33

IGR 1-2,582 36

IGR 1-2,1134 35

IGR $3,873 \quad 35$

IGR 3,1020= CIL 3,184 35

IGR 3,1093 = Waddington $2562 \mathrm{~g} \quad 34$

IGR 3,1094 34

IGR 3,1199= Waddington 207533

IGR 3,1200a 33

IGR 3,1286 35

IGR 3,1363 33

IGR 4,592, $593 \quad 34$

IGR 4,1480 35

ILS $540 \quad 34$

ILS $556=$ CIL 9,5682 21

ILS $557=$ CIL 8,8473 21

ILS 55821

\begin{tabular}{|c|c|c|}
\hline ILS 559 & & \\
\hline ILS 579 & 21 & \\
\hline ILS 585 & 22 & \\
\hline ILS $593=$ & $=\mathrm{CIL} 2,1115 \mathrm{f}$. & 21 \\
\hline ILS 608 & 22 & \\
\hline ILS $609=$ & $=$ CIL 8,4221 & 22 \\
\hline ILS $618=$ & $=\mathrm{CIL} 3,5810$ & 45 \\
\hline ILS $621=$ & $=$ CIL 6,254 2 & 22 \\
\hline ILS $622=$ & $=$ CIL 6,255 2 & 22 \\
\hline ILS $629=$ & $=\mathrm{CIL} 3,710$ & 23,47 \\
\hline ILS 634 & 24 & \\
\hline ILS 671 & 27 & \\
\hline ILS $673=$ & $=$ CIL 6,1138 & 27 \\
\hline ILS 682 & 26 & \\
\hline ILS $692=$ & $=$ CIL 6,1140 & 28 \\
\hline ILS 693 & 28 & \\
\hline ILS $697=$ & $=$ CIL 5,8011 & 28 \\
\hline ILS $699=$ & $=$ CIL 11,9 29 & \\
\hline ILS 702 & 29 & \\
\hline ILS $732=$ & $=$ CIL 3,3705 & 28 \\
\hline ILS $758=$ & $=$ CIL 8,7014 & 25 \\
\hline ILS $779=$ & $=$ CIL 8,10489 & 25 \\
\hline ILS 4175 & 35 & \\
\hline ILS 4918 & $=$ CIL 10,3792 & 37 \\
\hline ILS 8881 & 42 & \\
\hline OGIS 723 & & \\
\hline
\end{tabular}

ILS $559 \quad 21$

ILS 58522

ILS $593=$ CIL 2,1115f. 21

22

ILS $618=$ CIL 3,5810 45

ILS $621=$ CIL 6,254 22

ILS $622=$ CIL 6,255 22

ILS $629=$ CIL 3,710 23, 47

ILS 63424

ILS $673=$ CIL 6,1138 27

ILS 68226

ILS $692=$ CIL 6,1140 28

ILS 69328

ILS $697=$ CIL 5,8011 28

ILS $699=$ CIL 11,9 29

ILS 70229

ILS $732=$ CIL 3,3705 28

ILS $758=$ CIL 8,7014 25

ILS $779=$ CIL 8,10489 25

ILS $4175-35$

ILS $8881 \quad 42$

OGIS 72337 


